\title{
The Design and Implementation for the Embedded Data Terminal of Chemical Defense Equipment
}

\author{
Xuezheng Zhu, Ding Wang, Yuguang Wang, Jin Gu, Zhirong Luo \\ Institute of Chemical defense, Beijing 1022205
}

\begin{abstract}
Keywords: Embedded system; Chemical protective equipment; Terminal; Collecting and storing; Date processing
\end{abstract}

\begin{abstract}
Based on the embedded system design scheme and pulled by the collection, transmission, processing and application requirements of the equipment information, this paper completed the design and implementation of software and hardware in data terminal of chemical protective equipment, which can collect, store and monitor the information of the equipment's self-check data, operational data, status data, failure data, alarm data, etc. in real time. Meanwhile, the tested accuracy of collecting, transmitting and storing some equipment's information can guide the activities of chemical protective equipment effectively such as improvement and training, which innovatively provided the subsequent application development with fundamental and standardized information base.
\end{abstract}

\section{Introduction}

The chemical protective equipments of large vehicles own complex structure and strong compound function, which have stronger dependency to professional ancillary equipment of inspection and maintenance in the process of training, managing and maintaining, so the corresponding personnel should own higher level. With the continuous development of the microprocessor, the application of embedded system in the equipment system has been greatly accelerated. The design and implementation of data terminal of chemical protective equipment has provided the information management of PC chemical protective equipment and the application of analysis system in equipment's maintenance with basic and standardized data, which is significant for enhancing the using efficiency of chemical protective equipment, reducing maintenance costs, and improving the efficiency of equipment management.

\section{System framework and its operating principle}

\section{System framework}

The embedded data terminal consists of signal conditioning module, data acquisition unit, embedded processor, data storage unit, bus communication unit and embedded processing software. The objects of its information collection mainly are operating information, such as radiometer's radiation and alarm data, biological alarming device's monitoring values of the concentration and temperature, etc., self-inspection information, such as reconnaissance equipment's probe testing data, decontamination equipment's voltage testing data, etc., status information, such as consumable apparatus of decontamination equipment's consumption, traffic mileage, continuous operating time of all the parts, temperature of working condition, etc., and fault information, such as part, time and code of fault.

In order not to affect the original signal of the chemical protective equipment in the process of monitoring and sampling information, the various kinds of interfaces of data terminal should be designed in accordance with the relevant standards. At the same time, the information acquisition methods and universal signal conditioning should be designed according to the characteristics of chemical protective equipment's information output, which guarantees the applicability, compatibility and extensibility of a variety of information sources. The overall design framework is shown in figure 1. 


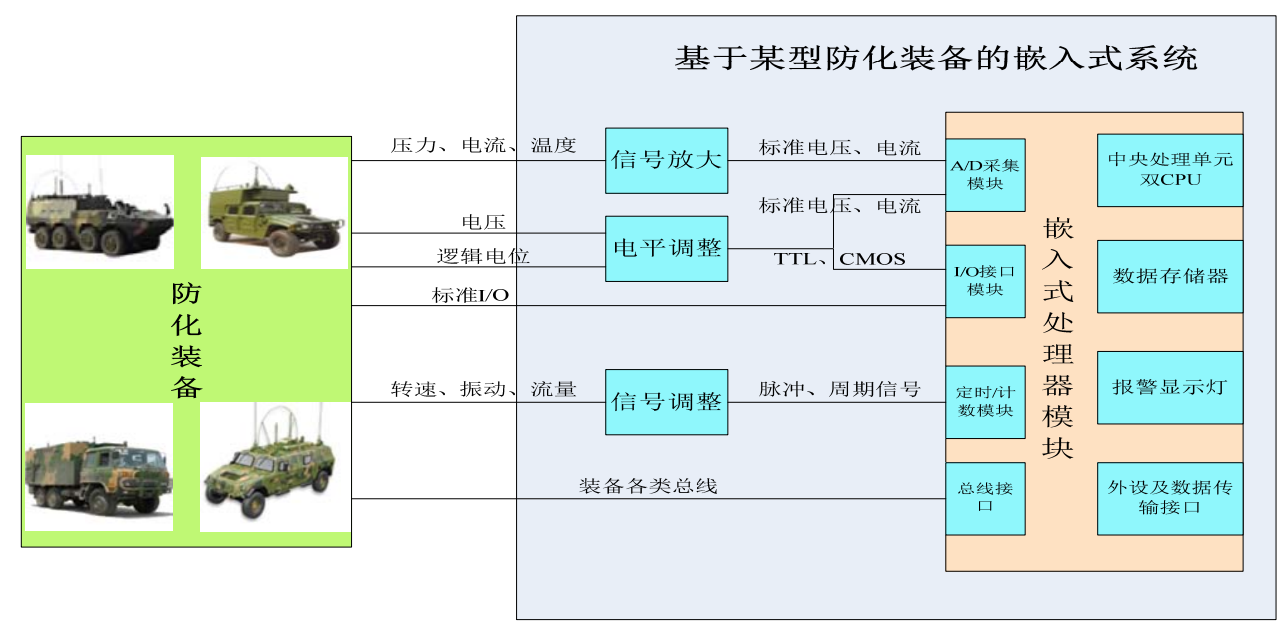

\section{Operating principle}

Figure 1 Design of embedded data terminal's whole frame

The information parameters' signal types of chemical protective equipment can be basically divided into the voltage, current, pressure, flow, temperature, speed and frequency, etc..The embedded data terminal, through connecting the control system or being equipped with sensors in the corresponding areas, can get information about equipment data by collecting bus signal of CAN, RS232 and RS485

\section{The design of hardware}

The design of the external structure

The design of the data terminal's front view and back view is shown in figure 2.
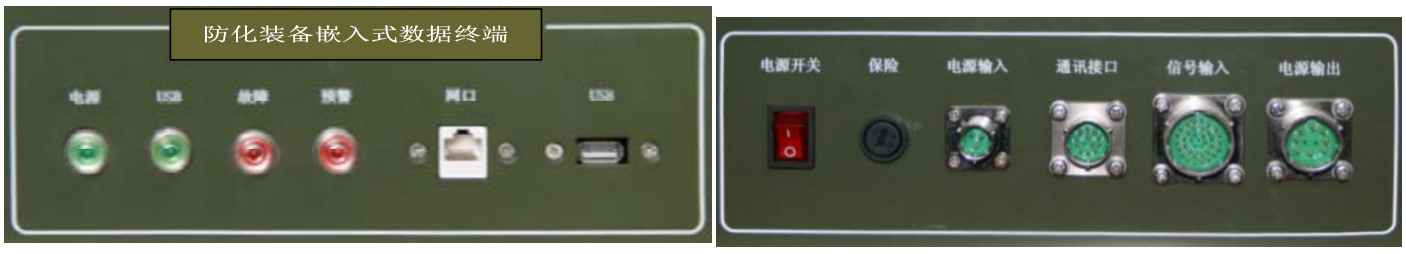

Figure 2 Front view and back view of the data terminal

\section{1) Illustration of front panel interface}

The front panel includes status indicators, internet access and USB interface. Status indicators show the information such as power status, fault indications and warning information; Internet access, connected to PC, can monitor the online operation and status information of the chemical protective equipments in real time; USB interface is connected to the USB flash disk or the upper machine to derive the stored information of data.

\section{2) Illustration of rear panel interface}

Rear panel includes power input interface, communication interface, signal input interface, power output interface, insurance and the power switch. Power input interface, connected to external power supply, provide $24 \mathrm{v}$ dc power for data terminals; Reserved power output interface can output de voltage, in order to supply power for the added sensor; Communication interface includes he bus of RS232 and RS485 and CAN, which is used for connecting to the control bus of chemical protective equipment; Signal input interface can be connected with the added sensor or reserved detection interface of chemical protective equipment, which is used for signal detection; Insurance is used to protect the internal circuit; Power switch is used to switch on or switch off the external power supply of data terminal.

\section{The design of internal structure}

The specific internal structure of data terminal is shown in figure 3. 


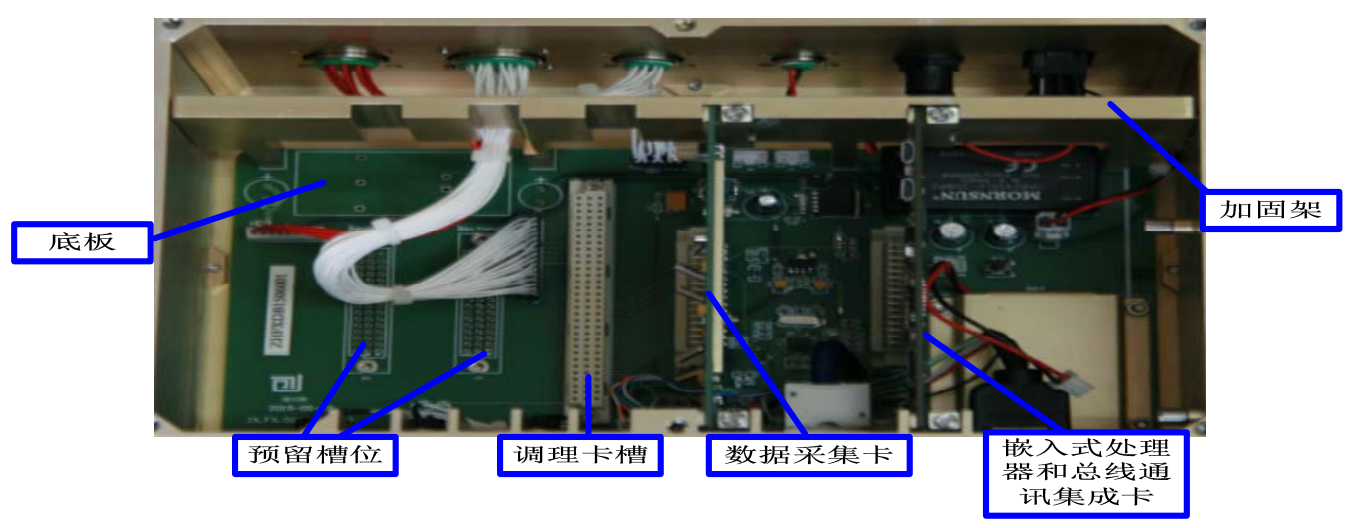

Figure 3 Internal structure of the data terminal

The internal structure adopts functional modular design and the modules are connected by specialized bus. The whole structure is made up by the backplane circuit, signal conditioning card, data acquisition card, bus communication card, reserved slot and embedded processor module, which can enhance the ability of function extension, maintenance and fault diagnosis of data terminal. The data acquisition card and embedded processor modules are connected by SPI bus; Bus communication card and embedded processor modules are connected by backplane communication bus (since this version of data terminal belongs to the prototype, which is mainly used for system testing, the bus communication card is integrated on the MCU board, but the improved version will have a separated card); reserved slot and embedded processor are connected by backplane $\mathrm{I}^{2} \mathrm{C}$ bus; According to its own resource definition, the embedded processor connects with the backplane; Each function module can be inserted and fixed from the reinforced frame on both sides according to the order from top to bottom.

\section{The design of modules}

\section{1) The module of signal conditioning}

The realization of these functions including analog signal conditioning, digital IO isolation and voltage level isolation can design the signal conditioning module into a functional board according to the functional group that is connected by specialized data bus connection backplane.

\section{a) Analog signal conditioning unit}

The external input analog signal should do the partial pressure to the range of AD collection, as the typical low pass filter circuit can finitely limited high-frequency interference signals, choose filter capacitance according to the tested signal frequency, and adjust the amplifier gain of circuit through adjusting feedback resistance, circuit is shown in figure 4.

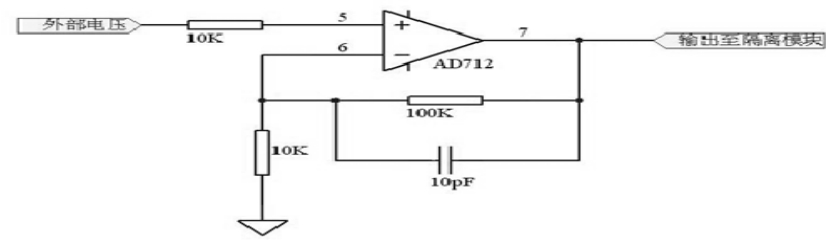

Figure 4 Input analog signal conditioning circuit

\section{b) Digital IO isolation unit}

Through the optical coupling isolation, the digital IO isolation unit converts the input signal of different amplitudes to TTL level signal that will be adjusted to be compatible with unit under test to protect the acquisition module of digital IO interface as well as enhance the driving capacity of the digital IO signals drive, circuit is shown in figure 5.
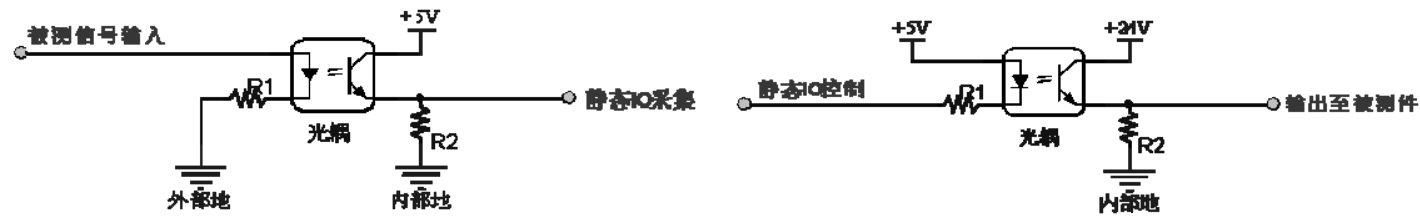

Figure 5 Isolation circuit of input and output signals 


\section{c) Voltage level isolation unit}

The voltage level isolation unit can realize the isolation, transformation and amplifier of dc voltage signal. It adopts the hybrid circuit of magneto-electric isolation and the input signal enters analog signal isolation amplifier through being filtered by the low-pass filter, which makes the interference suppression of analog signal ground and data isolation and collection into reality. It is easy to use and does not need null point and gain control, circuit is shown in figure 6.

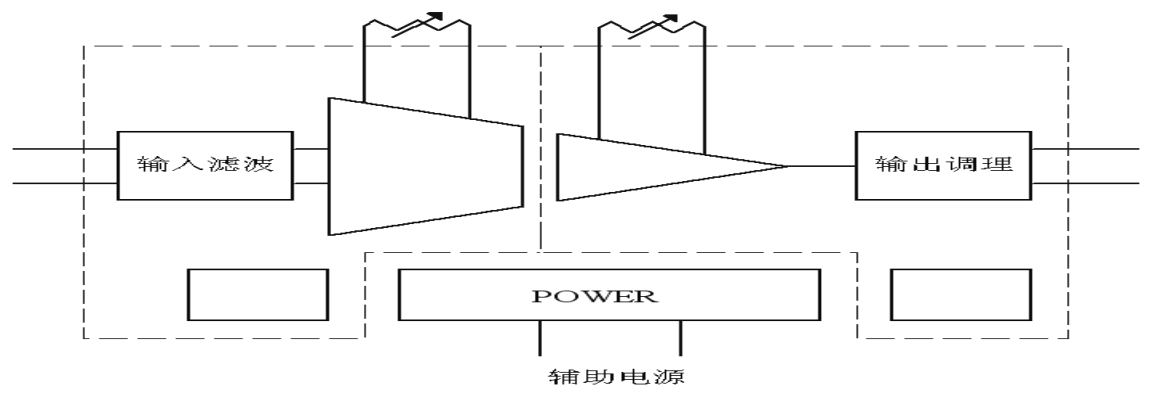

Figure 6 Circuit of voltage isolation module

\section{2) Data acquisition module unit}

The data acquisition module unit changes the conditioned analog signals into digital signals, which will be transmitted to the processor module for identification processing. In order to meet the sampling precision and testing demand, the data acquisition module adopts high-precision analog front end and the ADC acquisition chip, whose conversion accuracy can reach 16 and maximum sampling rate of single channel can be up to $500 \mathrm{KHZ}$. The processor communicates through SPI bus system and analog-to-digital conversion chip in serial way to get the sensor data. The relationship of the data flow is shown in figure 7.

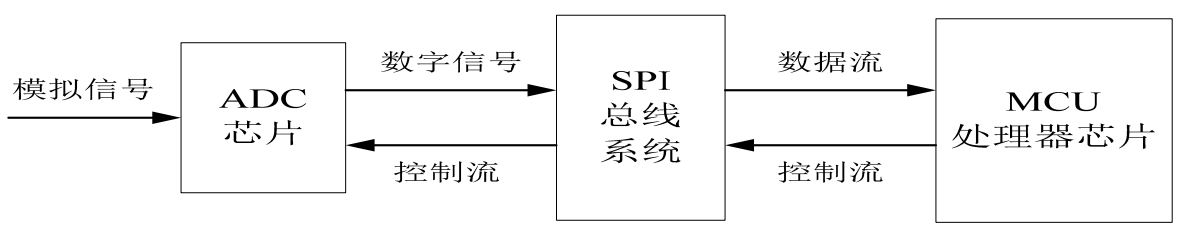

Figure 7 Schematic diagram of data acquisition

\section{3) Embedded processor module unit}

According to the requirements of data terminal function and environment, the embedded processor module chooses i.MX 6Quad application processor of Freescale based on ARM tioCortex - A9 gainestown; Its single frequency is 1GHz;Its internal storage of DDR3 is $1 \mathrm{~GB}$ and second-level cache is $1 \mathrm{MB}$; Multi-channel communication port can meet the demand of information transmission and sustain f HDMI1.4、USB2.0 and kilomega ethernet card; The working temperature range is from- $25{ }^{\circ} \mathrm{C}$ to $45{ }^{\circ} \mathrm{C}$ and storage temperature range is from $-40{ }^{\circ} \mathrm{C}$ to $80{ }^{\circ} \mathrm{C}$, which can meet the request of environment adaptability.

\section{4) Information storage module unit}

Considering factors such as information storage rate, capacity and cost, the information storage chooses 8GB Flash memorizer can meet the demand of storage, which will be integrated into the embedded processor module directly

\section{5) Bus communication module}

The RS232, RS485 and CAN bus communication functions are integrated into a functional board, which is connected to the embedded processor module through the backplane communication bus, and it can be added and tailored flexibly according to demands. In addition, in order to meet the test requirements of the CAN bus, the bus communication module designs the CAN bus communication interface circuit to support two ports to independently operate networks or bridge joint. The controller adopts SJA1000, so it has the function of bus arbitration and error detection, which can automatically correct the detected error and resend. 


\section{The design of software}

The embedded processing software includes the operating system and application software, which can complete the function of the chemical protective equipment's information collection and processing with the assistance of all the software modules and data flow.

\section{Description of development environment and function}

The embedded operating system chooses to use Linux/Android system kernel customized according to function requirements. The application software, developed based on ADS platform, is in charge of managing and controlling equipment's hardware.

Information acquisition software can realize that the embedded processors get the data information from the data acquisition card or bus communication card of chemical protective equipment though SPI or CAN bus according to certain frequency.

Data processing software converts the conditioned signal data to the corresponding physical quantities for calls of relevant procedure. Data judging software can analyze, compare and judge the obtained parameters data and set parameters threshold by the system. If the values are not in the normal range, determine equipment department components must be on fault and the judgment results will be transferred to fault alarm module.

Information storage software can classify and store the equipment information that has been collected, processed and judged to data storage unit according to the stated agreement format. If system components or sensor are on fault, the produced corresponding fault code as well as the information of equipment's state at failure moment will be stored into storage unit.

Fault alarm software can issue instructions according to the judgment results and controls the colors of indicator light in corresponding state. Data communication software can send the results of data processing to PC equipment information management and analysis system through the terminal internet access for real-time monitoring and analysis of the working parameters of chemical protective equipment.

Information output software can export the stored data information of chemical protective equipment to through the terminal USB port. Terminal processing software can receive the set command PC system terminal and response processing and feedback results.

\section{Data processing scheme}

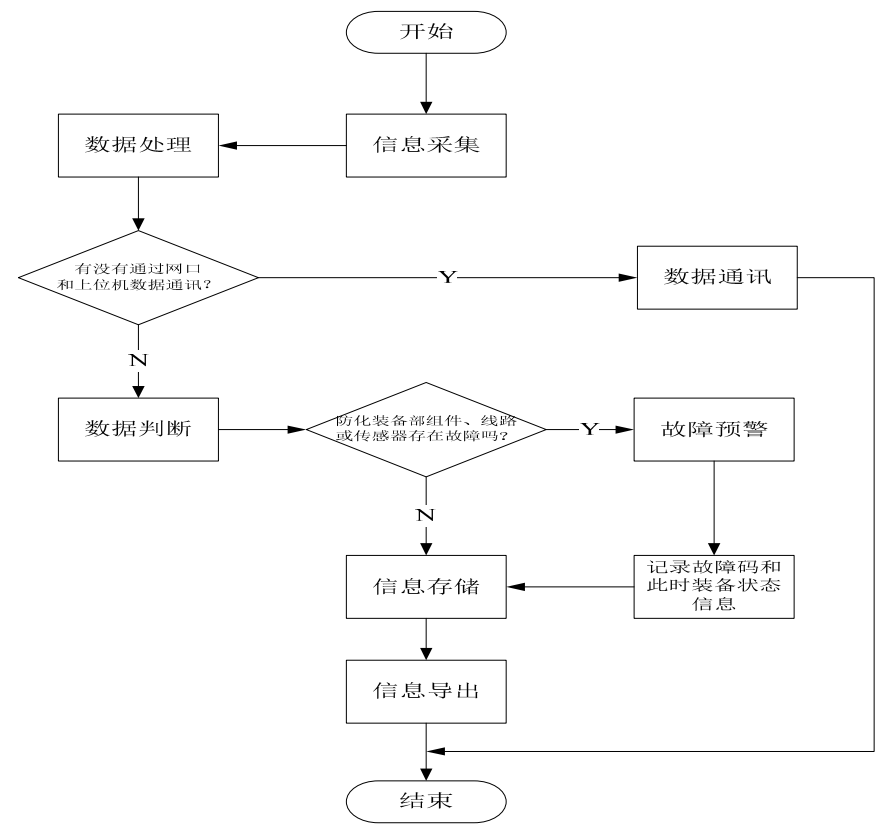

Figure 8 Data processing scheme

Embedded application software processes data signal by adopting the data flow. After that, it can judge whether the data communication exists in PC system, if not, it will do the data judgment. If some fault occurs, the alarm system will be started and store the fault code and current equipment state information, which can be exported when necessary. The data processing scheme of all the 
modules is shown in figure 8 .

\section{Experimental verification}

After the design is completed, the data terminal has been equipped in more than one type of chemical protective equipments for trial and part of its exported information of some equipment to the PC system is: Powering on time is PM.14:56, powering off time is PM.15:18, mixture flow is $2.1 \mathrm{~m}^{3} / \mathrm{h}$, accumulated hours is $312 \mathrm{~h}$, accumulated mileage is $3698 \mathrm{~km}$, speed of A pump is $2170 \mathrm{rpm}$,flow of B pump is $26 \mathrm{~L} / \mathrm{min}$, some unit pressure is $2.7 \mathrm{Mpa}$ and hydraulic oil temperature is $10{ }^{\circ} \mathrm{C}$.

Compared with the parameters information obtained by the equipment's control system, the data terminal can realize the data acquisition, data conversion, data analysis, classification, storage, and other functions of chemical protection equipment's related information. It can connect PC through the internet ports to on-line monitor parameters of all kinds of information, and can also connect PC via a USB to export stored equipment information for offline data processing and application analysis.

\section{Conclusion}

Based on the overall framework design, this paper has discussed the embedded data terminal design and implementation in detail from the aspects of hardware and software respectively. The experiments have proved that this terminal can obtain the information data of operation, training, management and maintenance in the process of using the chemical protective equipment, which has innovatively solved the problems such as non-standard record, no classification, artificial tamper, etc. It provides the PC equipment information management and analysis system with basic and standardized data sources, and affords the scientific decision basis to the chemical protective equipment training, which owns a broad application prospect.

\section{Reference}

[1] Song Jianshe, Cao Xiaoping, Cao Yaoqin, etc. Equipment Maintenance Information Engineering [M]. Beijing: national defence industry press, 2005.02

[2] Xue Yuan, Liu Weidong. Design and Implementation of a Multi-channel Real-time Data Acquisition System [J]. Computer measurement and control, 2011, 19 (4): 863-866

[3] Zhao Yue. The Design and Implementation of a Wireless Data Terminal Based on ARM [D]. Master degree thesis of Anhui University, 2013.12

[4] Xiong Jiaqi. Research of data acquisition and transmission terminal and its application in environmental monitoring[D]. Master Thesis of Zhejiang University, 2007.02

[5] Zhang Qiwen. ARM Embedded Modules and Integrated Systems Commonly Used Design Example[M]. Beijing: electronic industry press, 2007

[6] Rao Yuntao. Field Bus CAN Principle and Application Technology [M]. Beijing: University of aeronautics and astronautics press, 2003

[7] Zhang Quan. The Design and Implementation of Embedded Control System Platform [D]. Master thesis of Northwestern Polytechnical University, 2007.02

[8] Ye Lin, Yu Xinjie. Machinery and Eequipment Modern Maintenance Technology-- State maintenance [J]. Journal of mechanical science and technology, 1999, 18 (3): 457-458 